\section{CLINICAL CANCER MULTI-GENE MUTATION PROFILING OF SOLID TUMOURS BASED ON NEXT-GENERATION SEQUENCING (NGS)}

Mirette M. Saad, Mioara Gavrilla, Keith Byron, David Deam Chemical Pathology and Molecular Biology Departments, Healthscope Pathology, Clayton, Vic, Australia

Introduction: Genomic sequencing technologies have enabled identifõcation of actionable targets (e.g., $B R A F$ in melanoma, $E G F R$ in lung cancer) thus correlating with clinical parameters and facilitating treatment selection. We validated and implemented a somatic mutation gene panel, using NGS to test for genomic alterations within a tumour to help predict response to targeted therapy in cancer patients.

Methods: We analysed 576 solid tumours including colorectal (58\%), lung (29\%) and melanoma (13\%), referred to our laboratory by clinicians. Somatic mutations within 10 cancer related genes were identified using next generation sequencing (NGS) on an Illumina MiSeq instrument.

Results and Discussion: Our data showed that $46 \%$ of colorectal cancer biopsies harboured a $R A S$ mutation, while $15 \%$, $13 \%$ and $4 \%$ harboured a BRAF, PIK3CA or PTEN mutation, respectively. Somatic mutations of EGFR, KRAS and BRAF were detected in $12.5 \%, 24 \%$ and $3 \%$ of lung cancers, respectively; whereas, $36 \%$ of BRAF and $16 \%$ of NRAS mutations were identified in melanoma tumours. In some of the received tissue specimens no detected mutations were found, while others were either insufficient or degraded to carry out genetic testing.

Conclusion: We performed a comprehensive somatic mutation profile using NGS of the tumour genome from many cancer types. This information is useful in selecting appropriate gene targeted therapy for cancer patients. 